\title{
Characterisation of two quorum sensing systems in the endophytic Serratia plymuthica strain G3: differential control of motility and biofilm formation according to life-style
}

Xiaoguang Liu ${ }^{1 *}$, Jinli Jia ${ }^{1}$, Roman Popat ${ }^{2}$, Catherine A Ortori ${ }^{3}$, Jun Li ${ }^{1}$, Stephen P Diggle ${ }^{2}$, Kexiang Gao ${ }^{4}$, Miguel Cámara ${ }^{2 *}$

\begin{abstract}
Background: N-acylhomoserine lactone (AHL)-based quorum sensing (QS) systems have been described in many plant-associated Gram-negative bacteria to control certain beneficial phenotypic traits, such as production of biocontrol factors and plant growth promotion. However, the role of AHL-mediated signalling in the endophytic strains of plant-associated Serratia is still poorly understood. An endophytic Serratia sp. G3 with biocontrol potential and high levels of AHL signal production was isolated from the stems of wheat and the role of QS in this isolate was determined.
\end{abstract}

Results: Strain G3 classified as Serratia plymuthica based on $16 \mathrm{~S}$ rRNA was subjected to phylogenetic analysis. Using primers to conserved sequences of luxIR homologues from the Serratia genus, splIR and spsIR from the chromosome of strain G3 were cloned and sequenced. AHL profiles from strain G3 and Escherichia coli DH5 $\alpha$ expressing spll or sps/ from recombinant plasmids were identified by liquid chromatography-tandem mass spectrometry. This revealed that the most abundant AHL signals produced by Spll in E. coli were N-3-oxo-hexanoylhomoserine lactone (3-oxo-C6-HSL), N-3oxo-heptanoylhomoserine lactone (3-oxo-C7-HSL), N-3-hydroxy-hexanoylhomoserine lactone (3-hydroxy-C6-HSL), Nhexanoylhomoserine lactone (C6-HSL), and N-heptanoyl homoserine lactone (C7-HSL); whereas Spsl was primarily responsible for the synthesis of $\mathrm{N}$-butyrylhomoserine lactone (C4-HSL) and N-pentanoylhomoserine lactone (C5-HSL). Furthermore, a quorum quenching analysis by heterologous expression of the Bacillus A24 AiiA lactonase in strain G3 enabled the identification of the AHL-regulated biocontrol-related traits. Depletion of AHLs with this lactonase resulted in altered adhesion and biofilm formation using a microtiter plate assay and flow cells coupled with confocal laser scanning microscopy respectively. This was different from the closely related S. plymuthica strains $\mathrm{HRO}-\mathrm{C} 48$ and $\mathrm{RVH}$, where biofilm formation for both strains is AHL-independent. In addition, QS in G3 positively regulated antifungal activity, production of exoenzymes, but negatively regulated production of indol-3-acetic acid (IAA), which is in agreement with previous reports in strain HRO-C48. However, in contrast to HRO-C48, swimming motility was not controlled by AHL-mediated QS.

Conclusions: This is the first report of the characterisation of two AHL-based quorum sensing systems in the same isolate of the genus Serratia. Our results show that the QS network is involved in the global regulation of biocontrol-related traits in the endophytic strain G3. However, although free-living and endophytic S. plymuthica share some conservation on QS phenotypic regulation, the control of motility and biofilm formation seems to be strain-specific and possible linked to the life-style of this organism.

\footnotetext{
* Correspondence: xgliu66@yahoo.com; miguel.camara@nottingham.ac.uk

IInstitute of Life Sciences, Jiangsu University, Zhenjiang 212013, China

${ }^{2}$ School of Molecular Medical Sciences, Centre for Biomolecular Sciences,

University of Nottingham, Nottingham NG7 2RD, UK

Full list of author information is available at the end of the article
} 


\section{Background}

Endophytic bacteria reside within the living tissue of their host plants without substantively harming it [1]. They can be beneficial to their host by promoting plant growth or acting as biocontrol agents [2,3]. Serratia plymuthica is ubiquitously distributed in nature, and most frequently associated with plants. This organism has been isolated from the rhizosphere and the phyllosphere of various plants, as an endophyte from the endorhiza of potato $[4,5]$, or as a contaminant in a raw vegetable processing line $[6,7]$. Over the last two decades, S. plymuthica has received steadily increasing attention as a biocontrol agent for mainly fungal diseases. Based on the international approved German directive (TRBA 466), it is nowadays classified within the risk group 1 by the DSMZ (German Collection of Micro-organisms and Cell Cultures), indicating that the species does not pose a threat to human health [5].

Quorum-sensing (QS) plays a central role within a number of bacterial gene regulatory networks by controlling gene expression in a population-dependent manner with the aid of small diffusible signal molecules [8]. In Gram-negative bacteria, $N$-acylhomoserine lactones (AHLs) are the best described QS signal molecules. AHLs are made by LuxI homologues and, when they reach a critical threshold concentration, activate their cognate LuxR-type regulators which in turns induce or repress multiple gene expression. QS systems are involved in various physiological processes in bacteria, including bioluminescence, conjugation, symbiosis, virulence and biofilm formation [9]. Biofilms are increasingly recognized as the predominant form of bacterial growth in the environment [10]. Growth in a biofilm provides many advantages for bacteria, including enhanced resistance to environmental stresses, such as desiccation and antimicrobials, as well as to host defenses [11]. It has been well documented that a number of plant beneficial rhizobacteria employ AHLs as signal molecules to regulate biocontrol activities including the triggering of systemic resistance in host plants and the production of antifungal compounds [12-15]. The phenotypes regulated by AHLs in Serratia species are remarkably diverse and of profound biological and ecological significance. These include motility and biofilm formation, production of antibiotics, exoenzymes and butanediol fermentation, synthesis of the plant growth promoting auxin indole-3-acetic acid (IAA) and promotion of plant colonisation and biocontrol against several plant diseases [13-16]. However, the role of AHL-mediated QS system(s) in the endophytic strains of plant associated Serratia is less well understood.

Quorum quenching can been achieved by degrading AHL signal compounds using lactonases or acylases and has been shown to interfere with expression of AHL- regulated traits, such as virulence in Erwinia carotovora, Erwinia amylovora [17,18], Burkholderia thailandensis [19], Burkholderia cepacia complex [20], and Pseudomonas aeruginosa [21,22]; or biocontrol activity in rhizospheric Serratia plymuthica HRO-C48 [14]. AiiAdependent signal degradation is a particularly useful tool to study the impact of quorum sensing in Gramnegative bacteria having multiple AHL regulatory circuits without the need to make mutants in the different AHL synthase genes [21].

In this study we describe the initial characterisation of two AHL-mediated QS systems in the wheat stem endophyte Serratia plymuthica G3 [23]. Two luxIR homologue genes, splIR and spsIR were identified from this strain, their AHL profiles characterised and their role in biocontrol traits were determined. The results presented show that whilst the control of some biocontrol traits by AHLs is conserved in distinct S. plymuthica isolates, the regulation of motility and biofilm formation is strain specific and possibly linked to the original environment of the isolate. These results provide new insights into the regulation of beneficial interactions between endophytic Serratia strains, pathogens and host plants and will help with the understanding of the inconsistencies in their biocontrol performance.

\section{Methods}

\section{Microorganisms, media and growth conditions}

The bacterial, fungal strains and plasmids used in this study are listed in Table 1. S. plymuthica G3 was isolated from the stems of wheat (Triticum aestivum L.) in Taian, Shandong, China. A spontaneous mutant resistant to rifampicin was selected for further experiments. S. plymuthica G3, its derivatives and the biosensor Chromobacterium violaceum CV026 [24] were grown in $\mathrm{LB}$ medium at $28^{\circ} \mathrm{C}$ and stored at $-80^{\circ} \mathrm{C}$ in $25 \%$ glycerol. When required, antibiotics were added at final concentrations of $100 \mu \mathrm{g} / \mathrm{ml}$ for ampicillin, $100 \mu \mathrm{g} / \mathrm{ml}$ for carbenicillin, $40 \mu \mathrm{g} / \mathrm{ml}$ for rifampicin, and $25 \mu \mathrm{g} / \mathrm{ml}$ for tetracycline. All antibiotics were purchased from Sigma. The fungal isolate Cryphonectria parasitica was from the authors' laboratory collection and was routinely cultured on potato dextrose agar (PDA) (Difco) at $25^{\circ} \mathrm{C}$.

\section{DNA preparation and manipulations}

Standard methods were used for plasmid and genomic DNA isolation, restriction enzyme digestion, agarose gel electrophoresis, ligation, and transformation [25].

\section{Phylogenetic analysis}

To gain a better taxonomic understanding of the Serratia G3 isolate a $16 \mathrm{~S}$ rDNA-based phylogenetic tree was compiled using the neighbour-joining method of 
Table 1 Bacterial strains and plasmids used in this study

\begin{tabular}{|c|c|c|}
\hline Strain/Plasmid & Description & $\begin{array}{l}\text { Reference/ } \\
\text { source }\end{array}$ \\
\hline \multicolumn{3}{|l|}{ Bacterial strain } \\
\hline Serratia sp. G3 & Wild type, Rif ${ }^{r}$ & This work \\
\hline G3/pME6000 & $\begin{array}{l}\text { G3 derivative transformed with the } \\
\text { pME6000 vector plasmid }\end{array}$ & This work \\
\hline G3/pME6863-aiiA & $\begin{array}{l}\text { G3 derivative transformed with the } \\
\text { pME6863 plasmid }\end{array}$ & This work \\
\hline $\begin{array}{l}\text { Chromobacterium } \\
\text { violaceum CV026 }\end{array}$ & $\begin{array}{l}\text { Violacein production-based AHL } \\
\text { bioreporter }\end{array}$ & 24 \\
\hline E. coli DH5a & $\begin{array}{l}\text { F- recA1 endA1 hsdR17 deoR thi-1 } \\
\text { supE44 gyrA96 relA1 D(lacZYA } \pm \text { argF) } \\
\text { U169 } k \text { - [u80dlacZDM15] }\end{array}$ & 25 \\
\hline E. coli S17-1 & $\begin{array}{l}\text { thi pro hsdR recA; chromosomal RP4; } \\
\mathrm{Tra}^{+} ; \mathrm{Sm} / \mathrm{Sp}^{\mathrm{r}}\end{array}$ & 25 \\
\hline \multicolumn{3}{|l|}{ Plasmid } \\
\hline pME6000 & Broad-host-range cloning vector; $T c^{r}$ & 21 \\
\hline pME6863 & $\begin{array}{l}\text { pME6000 carrying the aiiA gene of } \\
\text { strain A24 under the control of } \\
\text { constitutive lac promoter; } \mathrm{TC}^{r}\end{array}$ & 21 \\
\hline pUCP18::gfpmut3.1 & pUCP18 carrying gfpmut3.1 gene; $\mathrm{Cb}^{r}$ & 28 \\
\hline \multicolumn{3}{|c|}{ Fungal strain } \\
\hline $\begin{array}{l}\text { Cryphonectria } \\
\text { parasítica }\end{array}$ & & $\begin{array}{l}\text { Lab } \\
\text { collection }\end{array}$ \\
\hline
\end{tabular}

MEGA 4. The $16 \mathrm{~S}$ rRNA gene sequence from the G3 isolate, we recently published elsewhere [23] was analysed together with those from other members of the genus Serratia, including the S. plymuthica DSM 4540 type strain as a reference and the related strains S. proteamaculans DSM 4543, S. ficaria DSM 4569, S. entomophila DSM 12358, S. odorifera DSM 4582, S. marcescens DSM 30121, as well as S. plymuthica RVH1 from a raw vegetable processing line and an endophytic strain JA05 isolated from ginseng plants. In addition, Escherichia coli ATCC 25922 as an outgroup. These 16S rRNA sequences were obtained from GenBank. The tree topology was tested by bootstrap analysis of 1000 samplings.

\section{Cloning and sequencing of two pairs of LuxIR homologues from S. plymuthica strain G3}

Production of AHL signal molecules in strain G3 was detected using a T-streak assay with $C$. violaceum CV026 on plates. The following two pairs of primers for the cloning the splIR and spsRI loci were designed to the conserved regions of the corresponding genes in the genus Serratia using the ClustalW multiple sequence alignment program: SplIR-F: 5'-TTTGTAGAATACCGGCAA GCTGTT -3' and SplIR-R: 5'-CAGATCGTCACGGAGCCTGT-3'; SpsRI-F:5'-GAGAGGGTTCAGTGTC AAAT-3' and SpsRI-R: 5'-CCATGGAAGATGTAGA AATG-3'. These genes were amplified using G3 genomic DNA as a template by PCR and cloned into pMD-19T
(Takara, Dalian, China). The clones expressed the AHL synthases SplI or SpsI in E. coli DH5 $\alpha$ were selected by T-streak with $C$. violaceum $\mathrm{CV} 026$ for further identification of AHL profiles, and confirmed by PCR and sequencing (Sangon Co. Ltd., Shanghai, China). A neighbour-joining tree of LuxI family members was produced using the MEGA 4. Amino acid sequences of SplI and SpsI from the G3 isolate were aligned and analysed together with LuxI homologs from other eight members of Serratia and EsaI from Pantoea stewartii DC283. TraI of Agrobacterium vitis S4 was tested as outgroup. These amino acid sequences of LuxI homologs were obtained from GenBank. Confidence in neighbour-joining tree was determined by analysing 1000 bootstrap replicates.

\section{AHL degradation by heterologous expression of the AiiA acyl-homoserine lactonase}

A quorum-quenching approach was used to identify AHL-regulated biocontrol-related phenotypes in the endophytic strain G3. E. coli S17-1/pME6863 carrying the AHL-lactonase aiiA from the Bacillus sp. strain A24 under the control of the constitutive lac promotor [21] was used to mobilise aiiA into $\mathrm{G} 3$ by conjugation to obtain G3/pME6863-aiiA. G3 containing pME6000 was used as a control. Transconjugants were selected on LB plates containing $25 \mu \mathrm{g} / \mathrm{ml}$ of tetracycline and $40 \mu \mathrm{g} / \mathrm{ml}$ of rifampicin. Inactivation of the AHLs produced by strain G3 was evaluated by $\mathrm{T}$-streak with the C. violaceum CV026 biosensor strain and further confirmed by LC-MS/ MS analysis as described below.

\section{Extraction of AHLs from culture supernatants}

For extraction of signal molecules, all tested bacteria were grown in $10 \mathrm{ml}$ of $\mathrm{LB}$ overnight at $28^{\circ} \mathrm{C}$ with shaking. Cell-free culture supernatants (sterilized by passing through a $0.2-\mu \mathrm{m}$ pore filter) were extracted twice with equal volumes of ethyl acetate after which the extracted organic phases were pooled. The solvent was removed under vacuum and the resulting extract reconstituted in acetonitrile prior to LC-MS/MS analysis.

\section{Identification of AHL profiles by LC-MS/MS}

AHLs were examined by LC-MS/MS in the Centre for Analytical Bioscience, School of Pharmacy, University of Nottingham, UK. Briefly, the mobile phase A (Aqueous) was $0.1 \%$ formic acid in water (Sigma, MS grade) and mobile phase B (Organic) $0.1 \%$ formic acid in acetonitrile (Fisher). Two Shimadzu LC-10ADvp pumps in binary mode were run at $0.45 \mathrm{ml} / \mathrm{min}$ using the gradients as follows: isocratic flow at $0 \%$ for $1 \mathrm{~min}$, linear gradient from 0 to $50 \% \mathrm{~B}$ in $1.5 \mathrm{~min}, 70$ to $99 \%$ until $5.5 \mathrm{~min}$, isocratic until $7.5 \mathrm{~min}$. The column was re-equilibrated for a further 4 min including subsequent injection cycle time. The autosampler was a Shimadzu SIL-HTc. 
The column, a Phenomenex Gemini C18 (5 u) $3 \times 15 \mathrm{~mm}$ was held at $50^{\circ} \mathrm{C}$ in a Shimadzu oven, model CTO-10Avp. The MS detector was a 4000 QTrap from Applied Biosytems. Specific analyses were monitored in a targeted multireaction monitoring (MRM) mode in which all specific source and collision cell parameters had been optimized. Generic parameters were: ion source voltage $5000 \mathrm{~V}$, source temperature $450^{\circ} \mathrm{C}$, the curtain, collision activated dissociation gas (CAD, $\mathrm{N}_{2}$ ), nebulizer gas (GS1) and heater gas (GS2) set at 20, 6, 30 and 15 psi respectively. The quadrupoles were set at unit resolution and specific precursor-product ion pair parameters were determined automatically using the quantitative optimization facility of Analyst 1.4.1. Subsequent ion trap scans (enhanced product ion, EPI) were triggered by ion counts in any one MRM channel rising above 5000 counts per scan (cps). During these EPI scans, the declustering potential was ramped from 15 to $35 \mathrm{~V}$ and the collision energy was ramped between 20 and $80 \mathrm{~V}$. Product ions were monitored in the range 80 to 330 , with a default fill time of 250 msec using dynamic fill time and a scan rate of $1000 \mathrm{Th} /$ sec. Relative quantification was performed by peak integration of the extracted ion chromatogram of the relevant MRM ion channel. The LC/MS system was controlled by the Analyst 1.4.1 software and data analysis was performed using the same in quantitative mode. A wide selection of synthetic AHLs with or without a 3-oxo or 3-hydroxy substitution and with acyl side-chain lengths ranging from $\mathrm{C} 4$ to $\mathrm{C} 14$ each at either $1 \mu \mathrm{M}$ or $5 \mu \mathrm{M}$ concentrations, for AHLs with even and odd carbon chain lengths respectively, were used as standards. AHLs were identified and confirmed by comparing both the elution time and the MS spectra of the peaks obtained with those of the standards.

\section{Antifungal activity in vitro}

The antagonistic activity of G3 and its derivatives G3/ pME6863-aiiA and G3/pME6000 were tested against the phytopathogenic fungus Cryphonectria parasitica, the causal agent of chestnut blight as previously described [13].

\section{Motility assays}

Minimal swim motility agar plates contained $10 \mathrm{~g} /$ liter tryptone, $5 \mathrm{~g} /$ liter $\mathrm{NaCl}$ and $0.3 \%$ (wt/vol) Bacto agar [26]. A $1 \mu$ volume of overnight seed cultures grown at $28^{\circ} \mathrm{C}$ were inoculated onto swim agar plates and incubated at $28^{\circ} \mathrm{C}$ for $16 \mathrm{~h}$.

\section{Adhesion assays}

Adhesion is considered to be the first step in the development of bacterial biofilm. Bacterial adhesion on abiotic surface was measured using polystyrene microtitre plates in triplicate as described by O'Toole and Kolter, 1998 [27] with a few modifications. Overnight bacterial cultures were inoculated into the wells of microtiter plates in $100 \mu \mathrm{l}$ of LB or M9 medium (final concentration of $\mathrm{OD}_{600}$ 0.02) without shaking and incubated at $30^{\circ} \mathrm{C}$ for 24,48 and $72 \mathrm{~h}$, respectively. At $24 \mathrm{~h}$ intervals, the cell densities were determined at $600 \mathrm{~nm}$, followed by quantification of adhesion. The medium was removed, and the cells were stained with $0.1 \%$ solution of crystal violet $(\mathrm{CV})$ at room temperature for $20 \mathrm{~min}$. The dye was then removed and the wells were washed four times. Bound dye CV was solubilized with $95 \%$ ethanol, and the absorbance was measured at $570 \mathrm{~nm}$.

\section{Flow cell biofilm assays}

Firstly the strains G3/pME6863-aiiA and the vector control G3/pME6000 were tagged with the green fluorescent protein, GFP by electroporation with plasmid pUCP18::gfpmut3.1 [28]. The transconjugants were selected on LB plates supplemented with both tetracycline and carbenicillin, and verified through observation under the fluorescence microscope. Biofilms were cultivated in a modified flow chamber in $\times 20$ diluted LB. $100 \mu \mathrm{l}$ of bacterial overnight cultures $\left(\mathrm{OD}_{600}=0.1\right)$ were injected into each channel of flow cell and incubated at room temperature for 48 hours, at flow rate of $52.04 \mu \mathrm{l} / \mathrm{ml}$ for each channel.

\section{Capturing of confocal images}

Biofilms were visualized with an inverted Zeiss LSM700 microscope. The objective used was a Zeiss EC PlanNeofluar 10x/0.30. 6 replicate Z-Stacks, with an interval of $5.741 \mu \mathrm{m}$ and the pinhole at $1 \mathrm{AU}$, were acquired from each flow cell and used to create threedimensional representations of the biofilms. Biofilm structure was quantified from the $Z$ stacks using the image analysis software package COMSTAT [29].

\section{Production of exoenzymes, siderophores and indole-3- acetic acid (IAA)}

Proteolytic and chitinolytic activities and siderophores production were assayed as described previously [30,31]. HPLC (Agilent 1200LC) analysis of IAA production was performed as previously described [23,32].

\section{Statistical analysis}

All data were subjected to analysis of variance (ANOVA) using Fisher's least significant difference (LSD) and Duncan's multiple-range test to compare treatment mean values. Each trial was repeated at least twice with at least three replicates for each treatment.

\section{Nucleotide sequence accession numbers}

The GenBank accession numbers for the splIR, and spsRI genes from strain G3 are FJ919305 and FJ919306, respectively. 


\section{Results}

\section{Phylogenetic classification of S. plymuthica G3}

To classify phylogenetically the G3 strain isolated from wheat stems, the sequence from the 1474-bp fragment of $16 \mathrm{~S}$ rDNA from this isolate we previously determined (EU344964) [23] was subjected to phylogenetic analysis with different $16 \mathrm{~S}$ rDNA sequences from members of the genus Serratia and E. coli strain ATCC 25922 as the outgroup. The sequence alignment for the phylogenetic tree was constructed and evaluated with MEGA 4 using the neighbour-joining method (see Additional file 1). As the phylogenetic tree showed that the G3 isolate was clustered within the same group (confidence $=99 \%$ ) with RVH1 (AY394724) and the type strain DSM 4540 (AJ233433) of S. plymuthica, respectively. Therefore, Serratia sp. G3 was tentatively classified as $S$. plymuthica. It is worth noting that the atypical S. plymuthica RVH1 strain is unable to produce prodigiosin pigment when compared to the S. plymuthica DSM 4540 type strain, but a combined comparative analysis of $16 \mathrm{~S}$ rRNA and gyrB sequences, DNADNA hybridization, and biochemical characteristics unequivocally identified this strain as S. plymuthica [7].

\section{S. plymuthica G3 possesses two quorum sensing systems SplIR and SpsRI}

The homologues of the two LuxIR genes were cloned from strain G3 by PCR using primers against conserved sequences as described in the Material and Methods. PCR products of 1441-bp and 1391-bp corresponding to the expected $s p l I R$ and $s p s I R$ respectively were sequenced. The 1441 -bp resulting sequence included two open reading frames (ORFs) corresponding to the predicted AHL synthase gene splI (633-bp) and the response regulator gene splR (750-bp) (FJ919305). The splI and splR ORFs are convergent, overlapping by 29-bp in their 3 ' regions. The 1391-bp sequenced fragment carried two ORFs corresponding to the predicted AHL synthase gene spsI (687$\mathrm{bp})$ and the response regulator gene (spsR) (747-bp) (FJ919306). The spsR and spsI ORFs are also convergent and overlapping by 54-bp in their 3' regions.

Database searches using tblastx revealed that SplI (ACR22886) shares 99\% and 98\% identity to SplI (AAR32908, AAW27921) from S. plymuthica strains RVH1 and HRO-C48, respectively, as well as 83, 68, $67 \%$ identity to the SprI (AAK76733) from Serratia proteamaculans B5a, SpnI (AAN52498) from S. marcescens SS-1, and EsaI (AAA82096) from Pantoea stewartii DC283 respectively, which are mainly responsible for the synthesis of 3-oxo-C6-HSL [15,16,33-36]. The second LuxI homolog SpsI from G3 was most similar to the LuxI homolog (ABV39177) from the poplar endophytic bacterium S. proteamaculans 568 (86\% identity and 92\% similarity), then to SwrI (AAO38762) from
Serratia marcescens MG1 (74\% identity) and to SmaI (CAJ86499) from S. marcescens strain 12 (67\% identity), SmaI (CAB92553) from Serratia strain ATCC 39006 (60\% identity). The AHL synthases SwrI and SmaI catalyze preferentially the synthesis of C4-HSL and, in less amount, C6-HSL $[16,37,38]$. To examine the evolutionary relationship between the LuxI family members described above, a phylogenetic analysis was performed using MEGA 4 and the neighbour-joining tree was showed in Figure 1. The results were consistent with the similarity analysis of amino acid sequences within LuxI family members, the LuxI family synthases were clustered into two groups, and SplI and SpsI from strain G3 are classified into group A and group B, respectively.

\section{Spll and Spsl from S. plymuthica G3 produce multiple AHLs}

To determine which AHLs were made by each SplI and SpsI, LC-MS/MS analysis was performed on extracted culture supernatants from the wild type G3 strain as well as recombinant $E$. coli strains expressing splI or spsI and the spectra profiles compared to that of synthetic AHL standards. At least ten different AHLs were detected in varying abundance in the wild type G3, including unsubstituted AHLs (C4-HSL, C5-HSL, C6HSL, C7-HSL, C8-HSL), 3-oxo derivatives (3-oxo-C6HSL, 3-oxo-C7-HSL, 3-oxo-C8-HSL) and 3-hydroxy derivatives (3-hydroxy-C6-HSL, 3-hydroxy-C8-HSL). The most abundant and hence most likely biologically relevant AHLs detected in the spent culture supernatants of the endophytic strain G3 were 3-oxo-C6-HSL, C4-HSL, C6-HSL, 3-hydroxy-C6-HSL and 3-oxo-C7HSL. However, strain G3 did not produce long chain AHLs [23]. When expressed in E. coli (Table 2), the recombinant SplI produced all ten AHLs whereas SpsI produced only unsubstituted AHLs, including C4-HSL, C5-HSL, C6-HSL, C7-HSL, and C8-HSL. The most abundant one was C4-HSL from SpsI, 100 fold higher than that the production of this molecule by SplI in E. coli, suggesting that SpsI is could also be the main AHL synthase responsible for synthesis of this AHL in G3, in accordance with SwrI and SmaI from different S. marcescens strains $[37,38]$ which share similarity to SpsI. Both SpsI and SplI produce C6-HSL, but only SplI was responsible for the most abundant signal 3-oxo-C6HSL, that is similar to SplI from S. plymuthica strains HRO-C48 and RVH1 [14,32], SprI from S. proteamaculans B5a, SpnI from S. marcescens SS-1 [34,35], as well as EsaI from P. stewartii [36]. However, 3-oxo-C7-HSL and 3-hydroxy-C6-HSL have not been reported as the main AHL signals in other members of Serratia with the exception of S. plymuthica IC1270 which showed very weak production of the predicted 3-hydroxy-C6-HSL by TLC 


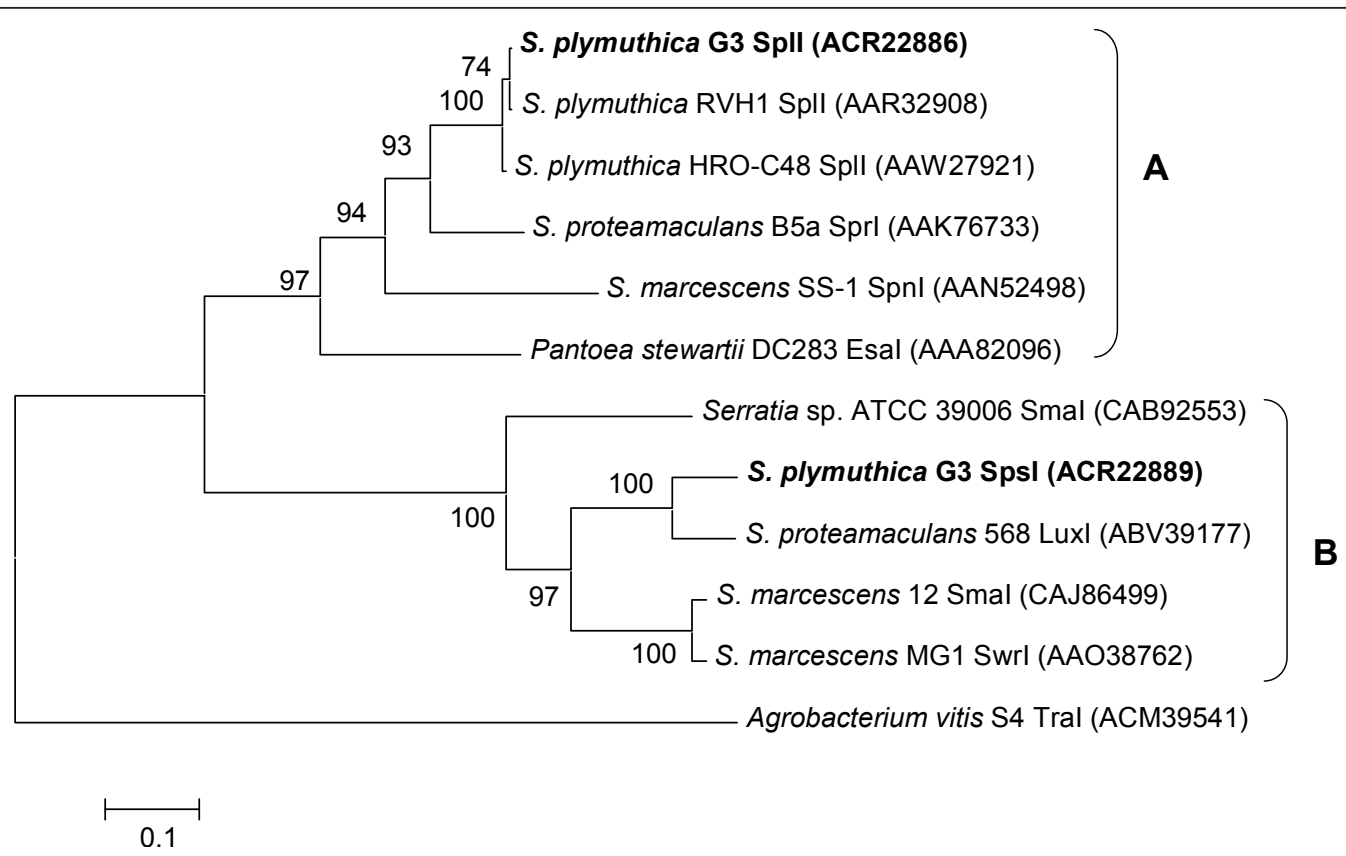

Figure 1 Neighbour-joining tree of Luxl family members in Serratia. The phylogenetic tree was generated using MEGA 4. Luxl family members in Serratia are clustered into two groups according to the AHL patterns. Spll and Spsl from G3 were in group A and group B, respectively. The significance of each branch is bootstrap value calculated for 1000 subsets. Scale bar indicates the mean number of substitutions per site.

analysis [30]. It is worth noting that there might be differences between AHL ratios from SplI and SpsI expressed in the wild type G3 and E. coli.

Heterologous expression of aiiA in G3 abolishes AHL accumulation and has an impact on biocontrol traits A number of bacteria are known to regulate various cell processes, including biocontrol activities through AHLmediated quorum sensing systems. To determine the

Table 2 AHL production by E. coli expressing either spll or spsl from $\mathbf{G 3}$

\begin{tabular}{ccc}
$\begin{array}{c}\text { AHL produced by G3 } \\
\text { WT[23] }\end{array}$ & $\begin{array}{c}\text { AHL expressed in } \\
\text { E. coli/splI }\end{array}$ & $\begin{array}{c}\text { AHL expressed in } \\
\text { E. coli/spsI }^{\#}\end{array}$ \\
\hline C4-HSL & + & ++++ \\
C5-HSL & + & +++ \\
C6-HSL & ++ & ++ \\
C7-HSL & ++ & + \\
C8-HSL & + & + \\
3-oxo-C6-HSL & +++ & - \\
3-oxo-C7-HSL & ++ & - \\
3-oxo-C8-HSL & + & - \\
3-hydroxy-C6-HSL & ++ & - \\
3-hydroxy-C8-HSL & + & - \\
\hline
\end{tabular}

AHL profiles identification was performed by LC-MS/MS from two independent experiments.

\# AHL mass abundance (relative quantity of íons from a particular AHL relative to that of a known standard) on LC-MS/MS: ++++ indicates $10^{7} ;+++$ indicates $10^{6} ;++$ indicates $10^{5} ;+$ indicates $\leq 10^{4}$. ability of the Bacillus A24 lactonase AiiA in degrading AHL signal molecules in G3, the plasmid pME6863aiiA, and the control vector pME6000 (lacking the aiiA gene) were introduced into the wild type G3 by mating with the E. coli donor strain S17-1. Overnight culture supernatants from these transconjugants were extracted in duplicate with solvent and subjected to LC-MS/MS semiquantitative analysis based on MRM mode showing that G3 harbouring the pME6000 vector control exhibited similar AHL patterns and concentration to the wild type (data not shown). In contrast, AHL production was practically abolished in G3 expressing aiiA from pME6863-aiiA (more than 99\% reduction), with only trace amounts of C4-HSL remaining which could not be detected by the biosensor CV026 and hence were unlikely to influence gene expression. This result suggested that AiiA can efficiently degrade all series of AHLs, including unsubstituted, 3-oxo, and 3-hydroxy at the third carbon position as it has been previously shown [39].

Impairment in AHL accumulation resulted in downregulation of the chitinolytic and proteolytic activities in G3/pME6863-aiiA. In contrast, biosynthesis of IAA increased five-fold and there was no effect on production of siderophores, compared to the wild type G3 and the control G3/pME6000 (see Additional file 2). This is in agreement with previous observations in S. plymuthica HRO-C48 heterologously expressing aiiA [14]. 
Swimming motility was also assayed to determine the role of quorum quenching by AiiA in motility. The swimming zones of the wild type G3, the AHL quenched strain G3/pME6863-aiiA and the vector control G3/pME6000 after incubation for $16 \mathrm{~h}$ at $28^{\circ} \mathrm{C}$ were $33.75 \pm 0.75 \mathrm{~mm}, 33.08 \pm 0.80 \mathrm{~mm}$, and $32.83 \pm 0.14$ $\mathrm{mm}$, respectively. The results suggest that, in contrast to S. plymuthica HRO-C48 where QS negatively regulates this phenotype [14], heterologous expression of aiiA in G3 has no influence on swimming motility (Figure 2).

\section{Lactonase expression in S. plymuthica G3 reduces antifungal activity in vitro}

Strain G3 exhibited inhibitory effects against several phytopathogenic fungal isolates in vitro and in vivo (data not shown). To determine the effect of quorum quenching by lactonase on antifungal activity, dual cultures were carried out, on single PDA plates, of the strain G3, G3/pME6863-aiiA or G3/pME6000 with C. parasitica, the cause of chestnut blight. After incubation for 4 days at $25^{\circ} \mathrm{C}$, the radius of the inhibition zones was measured. Although no large differences were observed between the wild type G3 and the control strain G3/pME6000, the radius of inhibition zones produced by G3/pME6863-aiiA was significantly decreased compared with the control G3/pME6000 and the wild type $\mathrm{G} 3$ at $P=0.01$ for $C$. parasitica (Table 3 .). The data showed that antifungal activity by G3 is partially dependent on AHL signaling via regulation of various exoenzymes and secondary metabolites.

\section{Abiotic surface adhesion and biofilm formation in $S$. plymuthica G3 are affected by lactonase expression} Many bacteria rely on QS systems to govern various aspects of biofilm development, including adhesion, motility, maturation, and dispersion [10,37]. Using microtiter plate assays, we evaluated the impact of quorum quenching by aiiA on adhesion to abiotic surfaces in G3. Figure 3A illustrates by $\mathrm{OD}_{600}$, there are no significant difference in bacterial growth rate between the wild type G3, G3/pME6000 and G3/pME6863-aiiA,

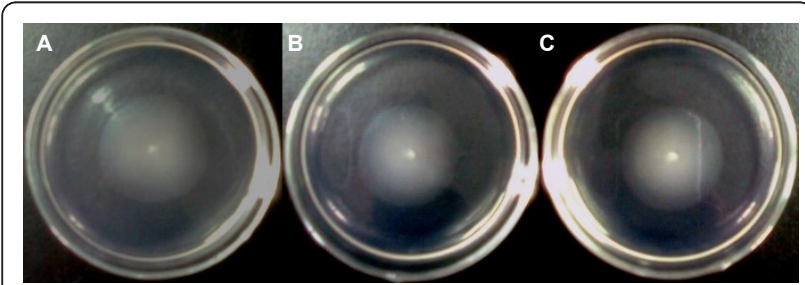

Figure 2 Swimming motility by $\mathrm{G} 3$ is independent of AHL signalling. One microlitre of overnight cultures of the wild type $\mathrm{G} 3$ (A), the control G3/pME6000 (B) and G3/pME6863-aiiA (C) were inoculated onto swim agar plates and incubated at $28^{\circ} \mathrm{C}$ for $16 \mathrm{~h}$.
Table 3 Effect of quorum quenching on antifungal activity in vitro

\begin{tabular}{|c|c|c|c|}
\hline \multirow{2}{*}{$\begin{array}{l}\text { Phytopathogenic } \\
\text { fungus }\end{array}$} & \multicolumn{3}{|c|}{ Inhibition zone $(\mathrm{mm})^{*}$} \\
\hline & G3 (wt) & $\begin{array}{c}\text { G3/pME6863- } \\
\text { aiiA }\end{array}$ & $\begin{array}{c}\text { G3/ } \\
\text { pME6000 }\end{array}$ \\
\hline $\begin{array}{c}\text { Cryphonectria } \\
\text { parasitica }^{a}\end{array}$ & $8.25 \pm 0.42$ & $5.91 \pm 0.20(B)$ & $\begin{array}{c}8.33 \pm 0.51 \\
(\mathrm{~A})\end{array}$ \\
\hline
\end{tabular}

but the strain G3/pME6863-aiiA showed a significant reduction in adhesion, compared with the vector control strain G3/pME6000 and the wild type G3 (Figure 3B).

Furthermore, 48 hour flow cell cultures of GFP-tagged G3/pME6863-aiiA and G3/pME6000 were observed and quantified for biofilm formation using CLSM during two
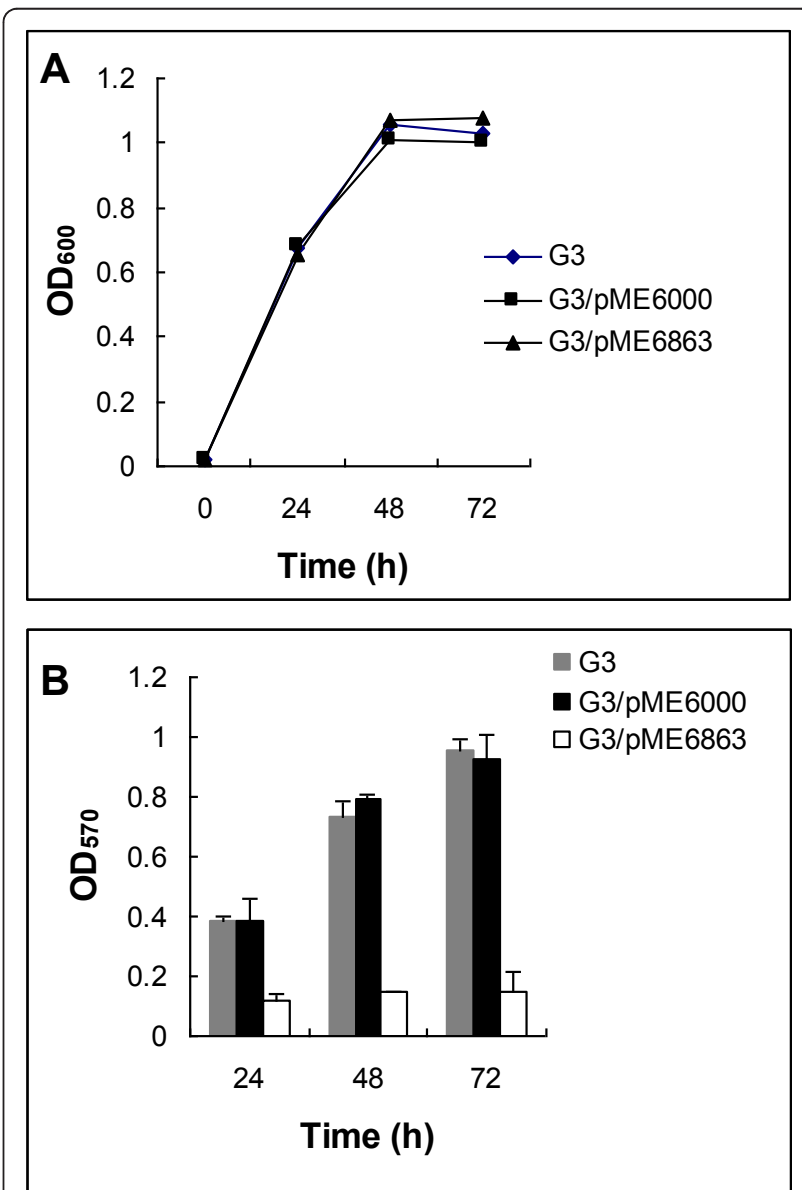

Figure 3 Effect of aiiA expression on abiotic surface adhesion by S. plymuthica G3. A: $\mathrm{OD}_{600}$ of $\mathrm{G} 3$ bacterial cultures in the presence and absence of the aiiA lactonase gene. B: Absorbance of crystal violet at $570 \mathrm{~nm}$ from stained cells bounds to polystyrene microtitre plate as a representation of adhesion. Experiments were done in triplicate. 
independent experiments. Figure 4A, B and 4C represent $2 \mathrm{D}$ optical slice and cross sections view (3D y and z-projection views) of bacterial biofilms developed after $24 \mathrm{~h}$ of growth by both strains. Quantitative analysis by COMSTAT indicated that not only the biofilm thickness (Figure
5A; the mean thickness of G3/pME6000::gfp and G3/ pME6863::gfp biofilms is $127.17 \pm 8.43 \mu \mathrm{m}$ and $32.10 \pm$ $5.10 \mu \mathrm{m}$ respectively), but also the biomass (Figure 5B; the biomass of G3/pME6000::gfp and G3/pME6863::gfp biofilms is $68.62 \pm 3.03 \mu \mathrm{m}^{3} / \mu \mathrm{m}^{2}$ and $12.63 \pm 1.39 \mu \mathrm{m}^{3} / \mu \mathrm{m}^{2}$
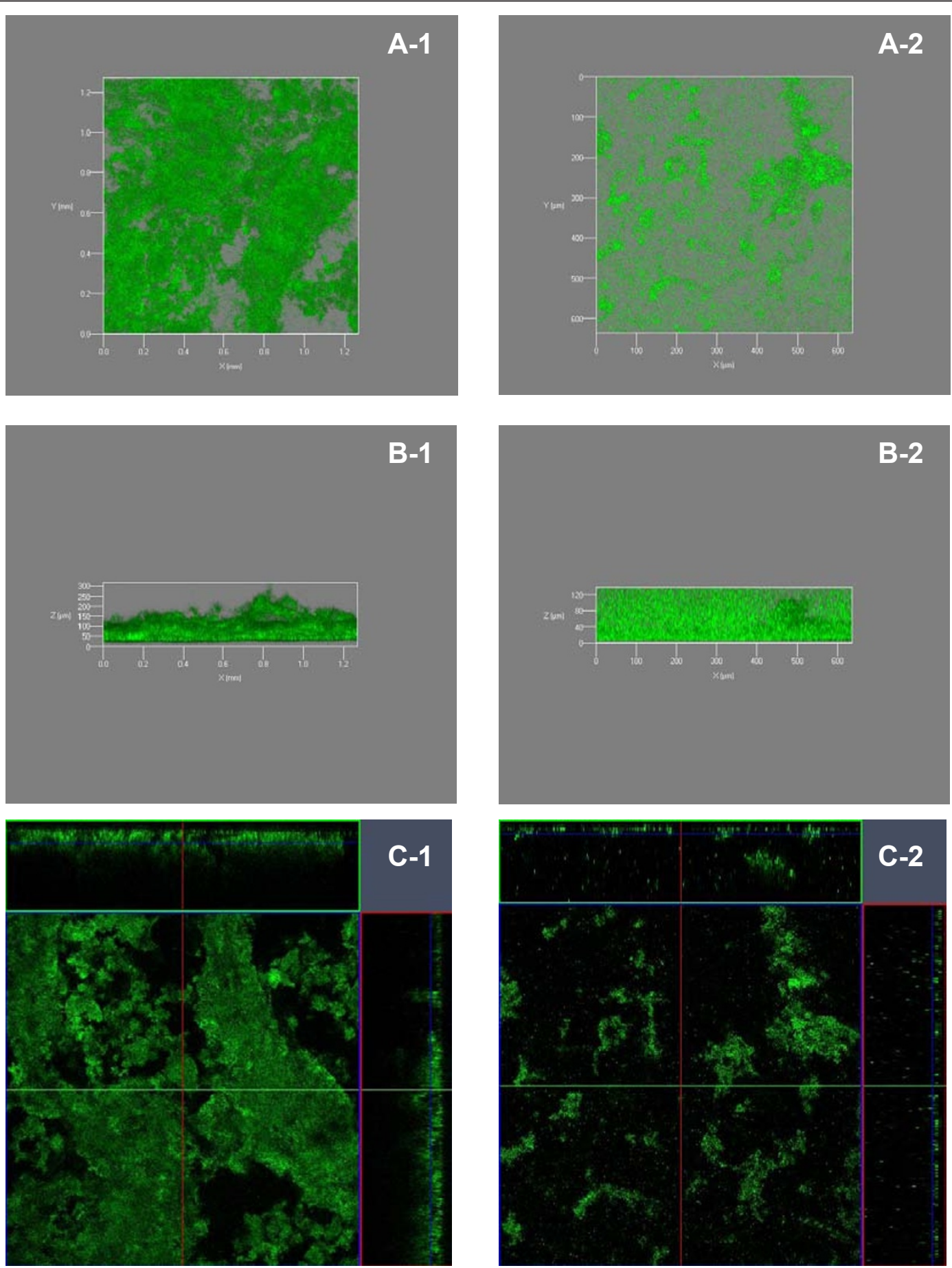

Figure 4 Effect of quorum quenching on biofilm formation. In vitro biofilm formation of the GFP-tagged strains G3/pME6000-pUCP18::gfpmut 3.1 (left panel) and G3/pME6863-pUCP18::gfpmut3.1 (right panel). Flow cell cultured biofilms incubated in 5\% LB were observed by confocal laser scanning microscopy after 48 h. A: 2 dimensional optical slice and cross sections, B: 3 dimensional y-projection; C: 3 dimensional z-projection. 

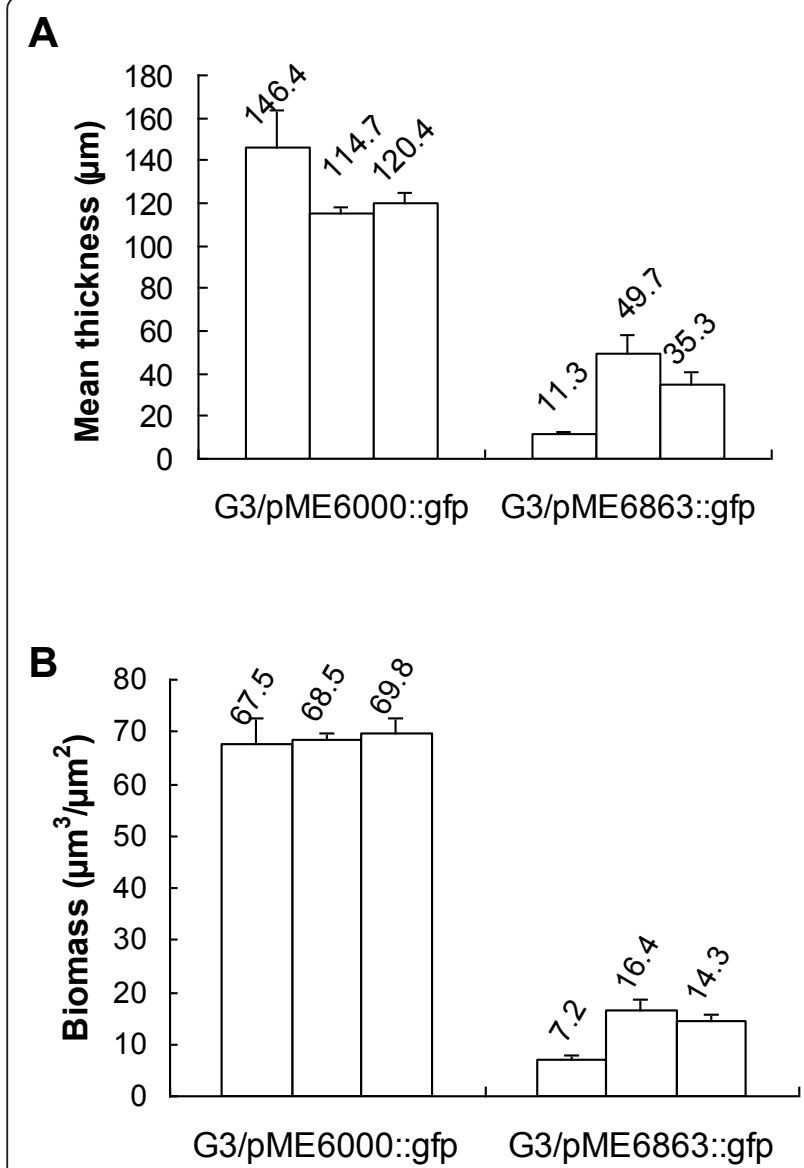

Figure 5 Quantitative analysis of the impact of aiiA expression on biofilm formation. The biofilm thickness (A) and the biomass (B) in flow cell were quantified by COMSTAT. Data represent mean \pm standard error of 6 random measurements with three independent channels.

respectively) between these two strains were significantly different, suggesting that biofilm development by G3, under the conditions used, is AHL-dependent.

\section{Discussion}

Endophytic bacteria have been found in virtually every plant studied, and there is increasing interest in developing their biotechnological potential to improve phytoremediation and the sustainable production of non-food crops for biomass and biofuel production [3]. In this manuscript we have reported that a new isolate of endophytic Serratia plymuthica G3 from the stems of wheat, exhibiting antifungal activities, produces high levels of AHLs and that the QS control of swimming motility and biofilm formation shows significant differences to other isolates of this organism from different environments previously described.

The ability of Serratia strains to produce AHLs and their AHL production profiles is well known to be species- and strain-dependent [16]. Previous works have also demonstrated that in S. marcescens SS-1 and S. plymuthica strains RVH1 and HRO-C48, SpnI or SplI knock out mutations abolished the production of 3-oxo-C6-HSL completely, but still retained residual AHL signals, suggesting the presence of additional AHL synthase(s) in some species of Serratia [15,33,35]. However, this is the first report showing the identification and initial characterisation of two QS systems splIR and spsIR in a single Serratia isolate. Sequence analysis showed that SplIR is highly similar to the SplIR of S. plymuthica strains RVH1 and HRO-C48, as well as SprIR of S. proteamaculans B5a and S. marcescens SS-1, all of which are responsible for the biosynthesis of 3-oxo-C6-HSL, and C6-HSL. Whereas SpsIR shares similarity to SwrIR and SmaIR from S. marcescens MG1 and strain 12 respectively, which are responsible for production of unsubstituted C4-HSL and smaller amounts of unsubstituted C6-HSL and C8-HSL (Table 2). In addition, these two sets of $l u x I$ and $l u x R$ homologous genes organized convergently in S. plymuthica G3 chromosome is characteristic of the most $\gamma$-proteobacteria $[33,35,40]$. The results were in line with the phylogenetic analysis (Figure 1), demonstrating that the LuxI family members from the genus of Serratia can be clustered into groups $\mathrm{A}$ and $\mathrm{B}$ according to the main AHL signals produced by bacteria, but it is not species-specific. For example, S. marcescens SS-1 was classified into group A as SplI of G3, known to produce 3-oxo-C6HSL. In contrast, Strain 12 and MG1 of S. marcescens were clustered into group B due to the production of C4-HSL as was SpsI from G3. Hence, our data provide new evidence to support that AHL patterns in Serratia is strain-dependent, indicating the presence of some conserved protein structure-function characteristics that would determine this specificity and which would be worth investigating in future. In addition, horizontal transfer of QS systems due to transposition or phagemediated events have been described for the spnIR locus of S. marcescens SS-1 and the smaIR locus from strain 12 to $274[16,38,41]$. Consequently, the presence of two QS systems in G3 may have originated from horizontal gene transfer amongst members of the genus Serratia. Gray and Garey (2001) also deduced that multiple LuxI and/or LuxR homologues present within single species have been usually acquired from independent sources [40].

Further comparative analysis of AHL profiles using LC-MS/MS from the wild type G3 and E. coli DH5 $\alpha$ expressing the recombinant plasmid carrying and splI or spsI showed that SplI is responsible for the synthesis of a broad range of AHLs with different substitutions whereas SpsI only drives the synthesis of AHLs with no substitutions on their acyl chains all of which are also 
made by SplI although some of them at much lower levels such as C4-HSL and C5-HSL. To our knowledge, the strain G3 is the only Serratia so far described with the ability to produce 3 different families of AHLs according to substitutions in position 3 (none, 3-oxo and 3-hydroxy), although this can be due to the improved LC-MS/MS techniques used with higher sensitivity to detect lower concentration and broader range of AHL signals. The most abundant AHL signals identified by LC-MS/MS from G3 were 3-oxo-C6-HSL and C4-HSL although significant levels of C6-HSL, 3-oxoC7-HSL and 3-hydroxy-C6-HSL were also detected [23]. However, the individual biological role of these AHLs remains unknown. Overlaps between the AHL profiles produced by different LuxI homologues in a single organism has been previously described in other bacteria such as Yersinia pseudotuberculosis [42] and this usually results in very complex QS regulatory cascades with a tight intraregulation between them [43]. Whether this level of complexity exists in the G3 strain remains to be elucidated.

In many plant beneficial rhizobacteria, QS mechanisms induce the synthesis of antimicrobial secondary metabolites and extracellular lytic enzymes with inhibitory effects towards other bacteria, fungi, protozoa, and nematodes [12]. The quorum quenching strategy using the lactonase AiiA was exploited to simultaneously quench the two AHL systems discovered in the endophytic strain G3 of S. plymuthica and investigate their role in controlling biocontrol-related phenotypes. The phenotypic analysis revealed that the strain G3/ pME6863 expressing aiiA had reduced antifungal activity, chitinolytic and proteolytic activities, but increased of IAA biosynthesis, and had no impact on siderophore production compared with the strain carrying the vector control G3/pME6000 and the wild type G3, indicating that QS control multiple biocontrol-related phenotypes in this strain. These results are in agreement with previous observations in the rhizospheric S. plymuthica HRO-C48 expressing AHL lactonases [14]. Depletion of AHLs with this lactonase resulted in altered adhesion and biofilm formation in vitro. This was different from the closely related S. plymuthica strains HRO-C48 and RVH1, where biofilm formation for both strains is AHLindependent. In addition, in contrast to $\mathrm{HRO}-\mathrm{C} 48$, swimming motility was not controlled by AHL-mediated QS [14,33].

Attachment is required for biofilm formation and these are key processes in the interaction between bacteria and plant tissues which have been shown to rely on quorum sensing [44]. For example, in the biocontrol bacterium Pseudomonas chlororaphis strain 30-84, QS systems and their control over phenazine production play a role in the successful formation of surface-attached populations required for biofilm formation. Transcriptome analysis revealed that phenazines as signals, upregulated many of the genes related to cell adhesion and biofilm development, such as fimbrial and lipopolysaccharides (LPS) genes [45]. The SwrIR quorum sensing system in $S$. marcescens MG1 plays a key role in biofilm development, from attachment to swarming motility, biofilm maturation and detachment, although QS regulation of adhesion in MG1 is surface dependent [37]. In S. marcescens strain 12, biofilm formation seems to rely on smaI, although this was measured using an attachment assay to a plastic microtitre plate [38], where SmaI is mainly responsible for C4-HSL synthesis. Pantoea stewartii causing Stewart's vascular wilt and leaf blight in sweet corn and maize utilizes the EsaI/EsaR QS system to control virulence and effective colonization. EsaI shares $80 \%$ similarity to SplI of G3 and is a typical AHL synthase that also catalyzes preferentially the synthesis of 3-oxo-C6-HSL. Both EsaI and EsaR mutants were not able to cycle through normal programmed bacterial development leading to the formation of atypical biofilms in vitro and loss of dissemination and infectivity in vivo, although the AHLdeficient strain displayed an unusually robust adhesion phenotype [36]. Under the experimental conditions used the ability of abiotic surface adhesion and biofilm formation by G3, using microtiter plate and flow cell assays respectively, is AHL-dependent, as the strain G3/ pME6863 expressed aiiA was impaired in these phenotypes in vitro. In contrast, previous studies based on microtitre plate assays reported that biofilm formation by the closely related S. plymuthica strains HRO-C48 and RVH1 were not affected by AHL signalling. This was demonstrated by the heterologous expression AiiA or the use of a splI-mutant in which 3-oxo-C6-HSL production was abolished, but still retained residual unsubstituted AHLs $[14,46]$. This suggests that QS may have different roles in S. plymuthica isolates from different environments.

A number of different factors might affect adhesion, including physicochemical interactions between the bacterium and the substratum, flagella, fimbriae, outer membrane proteins, and the presence of extracellular polymers. For instance, quorum-sensing regulation of adhesion, biofilm formation, and sloughing in S. marcescens MG1 has been shown to be surface dependent, and under the control of nutrient cues $[10,37]$. We predict that the variations on QS regulation of biofilm development among different strains of S. plymuthica is likely to be influenced by strainspecificity or their life style though this remains to be further investigated.

Consequently, this study reveals that, in S. plymuthica G3, QS positively controls antifungal activity, production of exoenzymes, but negatively regulated production of 
indol-3-acetic acid (IAA). This is in agreement with previous reports in strain $\mathrm{HRO}-\mathrm{C} 48$. However, in contrast to S. plymuthica strains HRO-C48 and RVH1, where biofilm formation is AHL-independent, in G3 adhesion and biofilm formation is controlled by QS. Finally, in contrast to $\mathrm{HRO}-\mathrm{C} 48$, swimming motility is not under QS control in G3 [14-16,33]. This work indicates the existence of a differential role for QS between endophytic and free living bacterial isolates suggesting that this regulatory mechanism can evolve to maximise the adaptation to different lifestyles.

\section{Conclusions}

Two QS systems SplIR and SpsIR from the endophytic S. plymuthica strain G3 have been characterised and their AHL profiles determined. This QS network is involved in global regulation of biocontrol-related traits, especially antifungal activity, adhesion and biofilm formation some of which are strain-specific in the Genus of Serratia. Further investigation will focus on the interplay between the two QS systems in strain G3 and the integration of QS into complex regulatory networks to modulate the beneficial plant-microbe interaction. This will ultimately lead to the optimisation of seed inoculums and provide novel strategies to improve the efficacy of S. plymuthica-mediated biocontrol and plant growth promotion.

\section{Additional material}

Additional file 1: Phylogenetic analysis of the $16 \mathrm{~S}$ rRNA gene sequences. The phylogenetic tree was produced using the 16S rRNA gene sequences corresponding to the endophytic strain $\mathrm{G} 3$ and other members of the genus Serratia. Escherichia coli ATCC 25922 was used as outgroup by the neighbour-joining method of MEGA 4. The significance of each branch is bootstrap value calculated for 1000 subsets. Scale bar indicates the mean number of substitutions per site. ${ }^{* T}$ Type strain.

Additional file 2: Heterologous expression of aiiA lactonases affects production of exoenzymes and secondary metabolites by $S$. plymuthica G3. Table showing the transconjugant strain G3/pME6863 reduced chitinolytic ( $48 \mathrm{~h}$ ) and proteolytic ( $48 \mathrm{~h}$ ) activities which played a key role in biocontrol activity, indicated by the smaller halo diameter, compared to the control G3/pME6000 and the wild type. However the biosynthetic level of auxin indole-3-acetic acid (IAA) was five times higher in G3/pME6863 $(2.77 \pm 0.01 \mathrm{\mu g} / \mathrm{ml})$ than in the control strain G3/ pME6000 $(0.57 \pm 0.01 \mu \mathrm{g} / \mathrm{ml})$ using HPLC analysis, when grown in LB supplemented with tryptophan for $48 \mathrm{~h}$ at $30^{\circ} \mathrm{C}$. Siderophore production measured at $36 \mathrm{~h}$ was $\mathrm{AHL}$-independent.

\section{Abbreviations}

AHL: N-acyl homoserine lactone; C4-HSL: N-butyrylhomoserine lactone; C5HSL: N-pentanoylhomoserine lactone; C6-HSL: N-hexanoylhomoserine lactone; C7-HSL: N-heptanoylhomoserine lactone; C8-HSL: Noctanoylhomoserine lactone; 3-oxo-C6-HSL: N-3-oxo-hexanoylhomoserine lactone; 3-oxo-C7-HSL: N-3-oxo-heptanoyl homoserine lactone; 3-oxo-C8-HSL: N-3-oxo-octanoylhomoserine lactone; 3-hydroxy-C6-HSL: N-3-hydroxyhexanoylhomoserine lactone; 3-hydroxy-C8-HSL: N-3- hydroxyoctanoylhomoserine lactone; BCA: Biocontrol agent; HPLC: High performance liquid chromatography; IAA: Indole-3-acetic acid; LC-MS/MS: Liquid chromatography-tandem mass spectrometry; QS: Quorum sensing;

\section{Acknowledgements}

This work was funded by the National Natural Science Foundation of China (NSFC) and the Royal Society of UK (RS) joint project (no. 30811130218); NSFC (30670030 and 30370954) and the funds from Jiangsu University (07JDG030, 07A005).

\section{Author details}

${ }^{1}$ Institute of Life Sciences, Jiangsu University, Zhenjiang 212013, China. ${ }^{2}$ School of Molecular Medical Sciences, Centre for Biomolecular Sciences, University of Nottingham, Nottingham NG7 2RD, UK. 'SChool of Pharmacy, University of Nottingham, Nottingham NG7 3RD, UK. ${ }^{4}$ Department of Plant Pathology, Shandong Agricultural University, Taian, China.

\section{Authors' contributions}

$\mathrm{XL}$ conceived and designed the study, carried out phylogenetic analysis, obtained GFP-tagged strains for biofilm analysis and wrote the paper; JJ, constructed strains of G3/pME6863-aiiA and G3/pME6000, and performed quorum quenching analysis of biocontrol phenotypes; RP, carried out flow cell biofilm assays and CLSM observation and images capture and statistical analysis; OCA, contributed to the LC-MS/MS identification of AHL profiles; JL, contributed to the AHL extracts and biofilm analysis; SPD, supervised biofilm assays and revised the manuscript; KG, contributed to the analysis of IAA and siderophores; MC, conceived and designed the study and critically revised the manuscript. All authors read and approved the final manuscript.

Received: 9 August 2010 Accepted: 1 February 2011

Published: 1 February 2011

\section{References}

1. Garafola C, Monchy S, Newman L, Hoffman A, Weyens N, Barac T, Vangronsveld J, van der Lelie D, Taghavi S: Genome survey and characterization of endophytic bacteria exhibiting a beneficial effect on growth and development of poplar trees. Appl Environ Microbiol 2009, 75(3):748-757.

2. Mercado-Blanco J, Bakker PAHM: Interactions between plants and beneficial Pseudomonas spp.: exploiting bacterial traits for crop protection. Antonie van Leeuwenhoek 2007, 92(4):367-389.

3. Ryan RP, Germaine K, Franks A, Ryan D, Dowling DN: Bacterial endophytes: recent developments and applications. FEMS Microbiol Lett 2008, 278(1):1-9.

4. Breed RS, Murray EGD, Hitchens AP: Bergey's manual of determinative bacteriology. 6 edition. Williams and Wilkins, Baltimore USA; 1948.

5. de Vleeschauwer D, Höfte M: Using Serratia plymuthica to control fungal pathogens of plant. $C A B$ Rev 2007, 2:046.

6. Van Houdt R, Aertsen A, Jansen A, Quintana AL, Michiels CW: Biofilm formation and cell-to-cell signalling in Gram-negative bacteria isolated from a food processing environment. J Appl Microbiol 2004, 96(1):177-184.

7. Van Houdt R, Moons P, Jansen A, Vanoirbeek K, Michiels CW: Genotypic and phenotypic characterization of a biofilm-forming Serratia plymuthica isolate from a raw vegetable processing line. FEMS Microbiol Lett 2005, 246(2):265-272.

8. Withers $H$, Swift $S$, Williams P: Quorum sensing as an integral component of gene regulatory networks. Curr Opin Microbiol 2001, 4(2):186-193.

9. Waters CM, Bassler BK: Quorum sensing: cell-to-cell communication in bacteria. Annu Rev Cell Dev Biol 2005, 21:319-346.

10. Rice SA, Koh KS, Queck SY, Labbate M, Lam KW, Kjelleberg S: Biofilm formation and sloughing in Serratia marcescens are controlled by quorum sensing and nutrient cues. J Bacterio/ 2005, 187(10):3477-3485.

11. Davies D: Understanding biofilm resistance to antibacterial agents. Nat Rev Drug Discov 2003, 2(2):114-122.

12. Dubuis C, Keel C, Haas D: Dialogues of root-colonizing biocontrol pseudomonads. Eur J Plant Pathol 2007, 119(3):311-328.

13. Pang Y, Liu X, Ma Y, Chernin L, Berg G, Gao K: Induction of systemic resistance, root colonization and biocontrol activities of the rhizospheric strain of Serratia plymuthica are dependent on $\mathrm{N}$-acyl homoserine lactones. Eur J Plant Pathol 2009, 124(2):261-268.

14. Müller H, Westendorf C, Leitner E, Chernin L, Riedel K, Schmidt S, Eberl L, Berg G: Quorum- sensing effects in the antagonistic rhizosphere 
bacterium Serratia plymuthica HRO-C48. FEMS Microbiol Ecol 2009, 67(3):468-478.

15. Liu X, Bimerew M, Ma Y, Muller H, Ovadis M, Eberl L, Berg G, Chernin L: Quorum- sensing signaling is required for production of the antibiotic pyrrolnitrin in a rhizospheric biocontrol strain of Serratia plymuthica. FEMS Microbiol Lett 2007, 270(2):299-305.

16. van Houdt R, Givskov M, Michiels CW: Quorum sensing in Serratia. FEMS Microbiol Rev 2007, 319(4):407-424.

17. Dong $Y H, X u J, L i X Z$, Zhang $L H$ : AiiA, an enzyme that inactivates the acylhomoserine lactone quorum-sensing signal and attenuates the virulence of Erwinia carotovora. Proc Natl Acad Sci USA 2000, 97(7):3526-3531.

18. Molina L, Rezzonico F, Défago G, Duffy B: Autoinduction in Erwinia amylovora: evidence of an acyl-homoserine lactone signal in the fire blight pathogen. J Bacteriol 2005, 187(9):3206-3213.

19. Ulrich RL: Quorum quenching: enzymatic disruption of $\mathrm{N}$-acylhomoserine lactone-mediated bacterial communication in Burkholderia thailandensis. Appl Environ Microbiol 2004, 70(10):6173-6180.

20. Wopperer J, Cardona ST, Huber B, Jacobi CA, Valvano MA, Eberl L: A quorum-quenching approach to investigate the conservation of quorum-sensing-regulated functions within the Burkholderia cepacia complex. Appl Environ Microbiol 2006, 72(2):1579-1587.

21. Reimmann C, Ginet N, Michel L, Keel C, Michaux P, Krishnapillai V, Zala M, Heurlier K, Triandafillu K, Harms H, Defago G, Haas D: Genetically programmed autoinducer destruction reduces virulence gene expression and swarming motility in Pseudomonas aeruginosa PAO1. Microbiol 2002, 148(4):923-932.

22. Sio CF, Otten LG, Cool RH, Diggle SP, Braun PG, Bos R, Daykin M, Cámara M, Williams P, Quax WJ: Quorum quenching by an N-acyl-homoserine lactone acylase from Pseudomonas aeruginosa PAO1. Infect Immun 2006, 74(3):1673-1682.

23. Liu X, Jia J, Atkinson S, Cámara M, Gao K, Li H, Cao J: Biocontrol potential of an endophytic Serratia sp. G3 and its mode of action. World J Microbiol Biotechnol 2010, 26(8):1465-1471.

24. McClean KH, Winson MK, Fish L, Taylor A, Chabra SR, Cámara M, Daykin M, Lamb JH, Swift S, Bycroft BW, Stewart GSAB, Williams P: Quorum sensing and Chromobacterium violaceum: exploitation of violacein production and inhibition for the detection of $\mathrm{N}$-acylhomoserine lactones. Microbiol 1997, 143(12):3703-3711.

25. Ausubel FM, Brent R, Kingston RE, Moore DD, Seidman JG, Smith JA, Struhl K: Current protocols in molecular biology John Wiley \& Sons Inc., New York, N.Y; 1994.

26. Atkinson S, Chang CY, Sockett RE, Cámara M, Williams P: Quorum sensing in Yersinia enterocolitica controls swimming and swarming motility. J Bacteriol 2006, 188(4):1451-1461.

27. OToole GA, Kolter R: Initiation of biofilm formation in Pseudomonas fluorescens WCS365 proceeds via multiple, convergent signalling pathways: a genetic analysis. Mol Microbiol 1998, 28(3):449-461.

28. Andersen JB, Sternberg C, Poulsen LK, Bjorn SP, Givskov M, Molin S: New unstable variants of green fluorescent protein for studies of transient gene expression in bacteria. Appl Environ Microbiol 1998, 64(6):2240-2246.

29. Heydorn A, Nielsen AT, Hentzer M, Sternberg C, Givskov M, Ersbøll BK, Molin S: Quantification of biofilm structures by the novel computer program COMSTAT. Microbiol 2000, 146(10):2395-2407.

30. Ovadis M, Liu X, Gavriel S, Ismailov Z, Chet I, Chernin L: The global regulator genes from biocontrol strain Serratia plymuthica IC1270: cloning, sequencing, and functional studies. J Bacteriol 2004, 186(15):4986-4993.

31. Schwyn B, Neilands JB: Universal chemical assay for the detection and determination of siderophores. Anal Biochem 1987, 160(1):47-56.

32. Crozier A, Arruda P, Jasmim JM, Monteiro AM, Sandber G: Analysis of indole-3-acetic acid and related indoles in culture medium from Azospirillum lipoferum and Azospirillum brasilense. Appl Environ Microbiol 1988, 54(11):2833-2837.

33. Van Houdt R, Moons P, Aertsen A, Jansen A, Vanoirbeek K, Daykin M, Williams $P$, Michiels CW: Characterization of luxl/luxR type quorum sensing system and $\mathrm{N}$-acyl homoserine lactone-dependent regulation of exo-enzyme and antibacterial component production in Serratia plymuthica RVH1. Res Microbiol 2007, 158(2):150-158.
34. Christensen AB, Riedel K, Eberl L, Flodgaard LR, Molin S, Gram L, Givskov M: Quorum-sensing-directed protein expression in Serratia proteamaculans B5a. Microbiol 2003, 149(2):471-483.

35. Horng YT, Deng SC, Daykin M, Soo PC, Wei JR, Luh KT, Ho SW, Swift S, Lai HC, Williams P: The LuxR family protein SpnR functions as a negative regulator of $\mathrm{N}$-acylhomoserine lactone-dependent quorum sensing in Serratia marcescens. Mol Microbiol 2002, 45(6):1655-1671.

36. Koutsoudis MD, Tsaltas D, Minogue TD, von Bodman SB: Quorum-sensing regulation governs bacterial adhesion, biofilm development, and host colonization in Pantoea stewartii subspecies stewartii. Proc Natl Acad Sci USA 2006, 103(15):5983-5988.

37. Labbate M, Zhu H, Thung L, Bandara R, Larsen MR, Willcox MD, Givskov M, Rice SA, Kjelleberg S: Quorum-sensing regulation of adhesion in Serratia marcescens MG1 is surface dependent. J Bacterio/ 2007, 189(7):2702-2711.

38. Coulthurst SJ, Williamson NR, Harris AK, Spring DR, Salmond GP: Metabolic and regulatory engineering of Serratia marcescens: mimicking phagemediated horizontal acquisition of antibiotic biosynthesis and quorumsensing capacities. Microbiol 2006, 152(7):1899-1911.

39. Wang L, Weng L, Dong Y, Zhang L: Specificity and Enzyme Kinetics of the Quorum- quenching N-Acyl Homoserine Lactone Lactonase (AHLlactonase). J Biol Chem 2004, 279(14):13645-13651.

40. Gray KM, Garey JR: The evolution of bacterial Luxl and LuxR quorum sensing regulators. Microbiol 2001, 147(8):2379-2387.

41. Wei JR, Lai HC: N-acylhomoserine lactone-dependent cell-to-cell communication and social behavior in the genus Serratia. Inter J Med Microbiol 2006, 296(2-3):117-124.

42. Ortori CA, Atkinson S, Chhabra SR, Cámara M, Williams P, Barrett A: Comprehensive profiling of $\mathrm{N}$-acylhomoserine lactones produced by Yersinia pseudotuberculosis using liquid chromatography coupled to hybrid quadrupole-linear ion trap mass spectrometry. Anal Bioanal Chem 2007, 387(2):497-511.

43. Atkinson S, Chang CY, Patrick HL, Buckley CM, Wang Y, Sockett RE, Cámara M, Williams P: Functional interplay between the Yersinia pseudotuberculosis YpsRl and YtbRl quorum sensing systems modulates swimming motility by controlling expression of flhDC and fliA. Mol Microbiol 2008, 69(1):137-151.

44. Danhorn T, Fuqua C: Biofilm formation by plant-associated bacteria. Ann Rev Microbiol 2007, 61:401-422.

45. Pierson LS, Pierson EA: Metabolism and function of phenazines in bacteria: impacts on the behavior of bacteria in the environment and biotechnological processes. Appl Microbiol Biotechnol 2010, 86(6):1659-1670.

46. Moons P, van Houdt R, Aertsen A, Vanoirbeek K, Engelborghs $Y$, Michiels CW: Role of quorum sensing and antimicrobial component production by Serratia plymuthica in formation of biofilms, including mixed biofilms with Escherichia coli. Appl Environ Microbiol 2006, 72(11):7294-7300.

doi:10.1186/1471-2180-11-26

Cite this article as: Liu et al: Characterisation of two quorum sensing systems in the endophytic Serratia plymuthica strain G3: differential control of motility and biofilm formation according to life-style. BMC Microbiology 2011 11:26.

\section{Submit your next manuscript to BioMed Central and take full advantage of:}

- Convenient online submission

- Thorough peer review

- No space constraints or color figure charges

- Immediate publication on acceptance

- Inclusion in PubMed, CAS, Scopus and Google Scholar

- Research which is freely available for redistribution

Submit your manuscript at www.biomedcentral.com/submit
C Biomed Central 